\title{
THE DUFFIN-SCHAEFFER CONJECTURE WITH EXTRA DIVERGENCE
}

\author{
ALAN HAYNES, ANDREW POLLINGTON AND SANJU VELANI
}

\begin{abstract}
Given a nonnegative function $\psi: \mathbb{N} \rightarrow \mathbb{R}$, let $W(\psi)$ denote the set of real numbers $x$ such that $|n x-a|<\psi(n)$ for infinitely many reduced rationals $a / n(n>0)$. A consequence of our main result is that $W(\psi)$ is of full Lebesgue measure if there exists an $\epsilon>0$ such that

$$
\sum_{n \in \mathbb{N}}\left(\frac{\psi(n)}{n}\right)^{1+\epsilon} \varphi(n)=\infty .
$$

The Duffin-Schaeffer Conjecture is the corresponding statement with $\epsilon=0$ and represents a fundamental unsolved problem in metric number theory. Another consequence is that $W(\psi)$ is of full Hausdorff dimension if the above sum with $\epsilon=0$ diverges; i.e. the dimension analogue of the Duffin-Schaeffer Conjecture is true.
\end{abstract}

Mathematics Subject Classification 2000: 11J83, 11K55, 11K60

\section{INTRODUCTION}

Throughout this note we will use the following standard notation from elementary number theory: $p$ denotes a prime number, $\mu(n)$ is the Möbius function, $\varphi(n)$ is the Euler phi function, $\omega(n)$ denotes the number of distinct prime divisors of $n$, and $\tau(n)$ is the number of positive integers which divide $n$. Also we use $\lambda$ to denote Lebesgue measure on $\mathbb{R} / \mathbb{Z}$ and $\operatorname{dim} X$ to denote the Hausdorff dimension of a set $X$.

Let $\psi: \mathbb{N} \rightarrow \mathbb{R}$ be a nonnegative arithmetical function and for each positive integer $n$ define $\mathcal{E}_{n} \subseteq \mathbb{R} / \mathbb{Z}$ by

$$
\mathcal{E}_{n}:=\bigcup_{\substack{a=1 \\(a, n)=1}}^{n}\left(\frac{a-\psi(n)}{n}, \frac{a+\psi(n)}{n}\right) .
$$

Denote the collection of points $x \in \mathbb{R} / \mathbb{Z}$ which fall in infinitely many of the sets $\mathcal{E}_{n}$ by $W(\psi)$. In other words,

$$
W(\psi):=\lim \sup _{n \rightarrow \infty} \mathcal{E}_{n}:=\bigcap_{m=1}^{\infty} \bigcup_{n \geq m} \mathcal{E}_{n} .
$$

AH: Research supported by EPSRC grant EP/F027028/1.

AP: Research supported by the NSF.

SV: Research supported by EPSRC grants EP/E061613/1 and EP/F027028/1. 
The famous Duffin-Schaeffer Conjecture [5] dates back to 1942 and is the assertion that $\lambda(W(\psi))=1$ if

$$
\sum_{n \in \mathbb{N}} \frac{\psi(n)}{n} \varphi(n)=\infty .
$$

Equivalently, the limsup set $W(\psi)$ is of full Lebesgue measure if the sum of the measures of the sets $\mathcal{E}_{n}$ diverges. Although various partial result 1 have been established (see [8] for details and references) the full conjecture represents one of the most difficult and profound unsolved problems in metric number theory. Our goal here is to prove a weaker statement in which 'extra divergence' is assumed. To this end, define the function $f:[0, \infty) \rightarrow \mathbb{R}$ by

$$
f(x):= \begin{cases}0 & \text { if } x=0, \\ x \cdot \exp \left\{\frac{\log x}{2 \log (-\log x)}\right\} & \text { if } 0<x<1, \\ 1 & \text { if } x \geq 1 .\end{cases}
$$

It is easily seen that as $x$ tends to zero from above, $f(x)$ tends to zero faster than $x(-\log x)^{-\epsilon}$ but more slowly than $x^{1+\epsilon}$ for any $\epsilon>0$.

Theorem 1. Let $\psi$ be any nonnegative arithmetical function and let $f$ be defined as above. Then $\lambda(W(\psi))=1$ if

$$
\sum_{n \in \mathbb{N}} f\left(\frac{\psi(n)}{n}\right) \varphi(n)=\infty .
$$

Clearly, (11) is valid whenever (2) is satisfied. In light of the comment preceding Theorem 1, we obtain the statement mentioned in the abstract as a special case.

Corollary 1. Let $\psi$ be any nonnegative arithmetical function. Then $\lambda(W(\psi))=1$ if there exists an $\epsilon>0$ such that

$$
\sum_{n \in \mathbb{N}}\left(\frac{\psi(n)}{n}\right)^{1+\epsilon} \varphi(n)=\infty
$$

As pointed out to us by Glyn Harman, Corollary 1 is equivalent to Theorem 3.7(iii) in his book [8]. We will say a little more about the connection to Harman's work in \$3, The following Hausdorff dimension statement is a consequence of Corollary 1 and the recent Mass Transference Principle [2] - see §5 for the corresponding Hausdorff measure consequence of Theorem 1,

Theorem 2. Let $\psi$ be any nonnegative arithmetical function. Then $\operatorname{dim}(W(\psi))=1$ if

$$
\sum_{n \in \mathbb{N}}\left(\frac{\psi(n)}{n}\right)^{1-\epsilon} \varphi(n)=\infty \quad \forall \epsilon>0 .
$$

\footnotetext{
${ }^{1} \mathrm{~A}$ partial result of particular importance is Khintchine's theorem from 1924. This fundamental theorem implies the Duffin-Schaeffer Conjecture under the assumption that $\psi$ is monotonic. This is hardly surprising since the conjecture is a direct result of attempting to remove monotonicity from Khintchine's theorem.
} 
It is easily verified that Theorem 2 implies the following result - see $\$ 4.2$ for the details.

Corollary 2. Let $\psi$ be any nonnegative arithmetical function. Then

$$
\operatorname{dim}(W(\psi))=1 \quad \text { if } \quad \sum_{n \in \mathbb{N}} \psi(n)=\infty .
$$

This result has previously been established by R. C. Baker and G. Harman - see Theorem 10.7 in [8]. Our approach is very different to theirs and enables us to prove the stronger statement given by Theorem 2 and more importantly pursue a natural line of questioning that 'converges' to the Duffin-Schaeffer Conjecture - see \$5. To compare the above dimension statements consider the function $\psi_{\tau}(n):=n^{-1}(\log n)^{-\tau}$ where $\tau>1$ is arbitrary. Regarding Theorem 2, it is readily verified that (4) is satisfied and so $\operatorname{dim}\left(W\left(\psi_{\tau}\right)\right)=1$. On the other hand, $\sum \psi_{\tau}(n)<\infty$ and so Corollary 2 is not applicable.

The following result is a direct consequence of Corollary 2 and the fact that

$$
\sum_{n \in \mathbb{N}} \frac{\psi(n)}{n} \varphi(n)=\infty \quad \Rightarrow \quad \sum_{n \in \mathbb{N}} \psi(n)=\infty .
$$

Corollary 3. Let $\psi$ be any nonnegative arithmetical function. Then

$$
\operatorname{dim}(W(\psi))=1 \quad \text { if } \quad \sum_{n \in \mathbb{N}} \frac{\psi(n)}{n} \varphi(n)=\infty .
$$

The corollary can naturally be interpreted as the dimension analogue of the Duffin-Schaeffer Conjecture.

\section{Preliminaries for Theorem 1}

A well known result due to Gallagher states that $\lambda(W(\psi))=0$ or 1 - see [8, Theorem 2.7]. Furthermore by the Borel-Cantelli Lemma from probability theory $\lambda(W(\psi))=0$ whenever the sum on the left of (11) is finite. If the sets in the collection $\left\{\mathcal{E}_{n}\right\}_{n \in \mathbb{N}}$ were pairwise independent then the divergence part of the same lemma would guarantee that $\lambda(W(\psi))=1$ whenever (11) is satisfied. However this turns out not to be the case and it appears that the best estimate of pairwise intersection is essentially the following result see [8, Lemma 2.8] and [9].

Lemma 1. Suppose $\psi$ is a nonnegative arithmetical function and for distinct $m, n \in \mathbb{N}$ let $d:=(m, n)$ and

$$
\Delta(m, n):=\max \left\{\frac{\psi(m)}{m}, \frac{\psi(n)}{n}\right\} .
$$

Then there exists a universal constant $c_{1}$ with the property that

$$
\lambda\left(\mathcal{E}_{m} \cap \mathcal{E}_{n}\right) \leq c_{1} \lambda\left(\mathcal{E}_{m}\right) \lambda\left(\mathcal{E}_{n}\right) P(m, n),
$$


where

$$
P(m, n):=\prod_{\substack{p \mid m n / d^{2} \\ p>m n \Delta(m, n) / d}}\left(1-\frac{1}{p}\right)^{-1} .
$$

The presence of the $P(m, n)$ term cannot be ignored, as it follows from one of Merten's theorems that

$$
\prod_{p \mid n}\left(1-\frac{1}{p}\right)^{-1} \gg \log \log n
$$

for infinitely many $n$. However it is not difficult to show that there exists a universal constant $c_{2}$ with the property that

$$
\prod_{\substack{p \mid n \\ p>\sqrt{\log n}}}\left(1-\frac{1}{p}\right)^{-1} \leq c_{2}
$$

for all $n$, so the only time when the $P(m, n)$ term can become large is when

$$
\frac{m n \Delta(m, n)}{d} \leq \sqrt{\log m n}
$$

It is also important to note that for $m \neq n$ if $\mathcal{E}_{m}$ intersects $\mathcal{E}_{n}$ then we must have that

$$
\left|\frac{a}{m}-\frac{b}{n}\right| \leq \frac{\psi(m)}{m}+\frac{\psi(n)}{n},
$$

for some integers $a$ and $b$ with $(a, m)=(b, n)=1$. Multiplying both sides of this equation by $m n$ reveals that

$$
\lambda\left(\mathcal{E}_{m} \cap \mathcal{E}_{n}\right)=0 \quad \text { unless } \quad d \leq \psi(m) n+\psi(n) m .
$$

It stands to reason that conditions (6) and (7) cannot be satisfied for a large proportion of pairs of integers $m$ and $n$. So although it is possible for two sets $\mathcal{E}_{m}$ and $\mathcal{E}_{n}$ to have some dependence we wish to show that this cannot happen on average at the same time that (2) is satisfied. The following well known tool from probability theory will then suffice to finish the proof - see [8, Lemma 2.3].

Lemma 2. Assume that (1) is satisfied. Then

$$
\lambda(W(\psi)) \geq \limsup _{N \rightarrow \infty}\left(\sum_{n=1}^{N} \lambda\left(\mathcal{E}_{n}\right)\right)^{2}\left(\sum_{m, n=1}^{N} \lambda\left(\mathcal{E}_{m} \cap \mathcal{E}_{n}\right)\right)^{-1} .
$$

Finally, we recall the following well known fact:

$$
\liminf _{n \rightarrow \infty} \frac{\varphi(n) \log \log n}{n}=e^{-\gamma} \quad \text { where } \gamma \text { is Euler's constant. }
$$

With all of these observations as a foundation we are ready to prove Theorem 1 . 


\section{Proof of Theorem 1}

Let $\psi$ be a nonnegative arithmetical function with support $S \subseteq \mathbb{N}$. By appealing to the Erdös-Vaaler Theorem [10] and to [9, Theorem 2] we will assume without loss of generality throughout the proof that $1 / n \leq \psi(n) \leq 1 / 2$ for all $n \in S$.

Our proof is divided into two main steps. In the first step we work with functions $\psi$ which are essentially constant or zero on long intervals of the form $\left[2^{3^{K}}, 2^{3^{K+1}}\right)$. This allows us to take full advantage of the inequalities (6) and (7) stated in the preliminaries. In the second step we show that we can deal with general functions $\psi$ by throwing away some of the support and thereby reducing the problem back to the 'constant' case. The extra divergence condition (2) plays a crucial role in both steps.

Step 1. For each nonnegative integer $k$ let

$$
\psi_{k}=\min \left\{\psi(n): n \in\left[2^{k}, 2^{k+1}\right) \cap S\right\} .
$$

To begin we will prove the theorem under the extra hypothesis that

$$
\max \left\{\psi(n): n \in\left[2^{3^{K}}, 2^{3^{K+1}}\right) \cap S\right\} \leq 2 \min \left\{\psi_{\ell}: 3^{K} \leq \ell<3^{K+1}\right\},
$$

for all $K \in \mathbb{N} \cup\{0\}$. Then it is easy to see that we have

$$
\sum_{n \leq 2^{K+1}} \lambda\left(\mathcal{E}_{n}\right)=\sum_{k=0}^{K} \sum_{n \in\left[2^{k}, 2^{k+1}\right)} \frac{\psi(n) \varphi(n)}{n} \gg \sum_{k=0}^{K} \frac{\psi_{k} \cdot\left|\left\{n \in S \cap\left[2^{k}, 2^{k+1}\right)\right\}\right|}{\log \log \left(2^{k}\right)} .
$$

Similarly hypothesis (2) guarantees that

$$
\sum_{k=0}^{\infty} 2^{k} f\left(\frac{\psi_{k}}{2^{k}}\right) \cdot\left|\left\{n \in S \cap\left[2^{k}, 2^{k+1}\right)\right\}\right|=\infty .
$$

This clearly ensures that

$$
\left|\left\{n \in S \cap\left[2^{k}, 2^{k+1}\right)\right\}\right|>\left(k^{2} 2^{k} f\left(\frac{\psi_{k}}{2^{k}}\right)\right)^{-1}
$$

for infinitely many $k$. Furthermore we may throw out all dyadic blocks for which (12) does not hold without affecting the divergence of (2). So without loss of generality we will now assume that $S$ has been chosen so that for every $k$ either (12) holds or else $\left|S \cap\left[2^{k}, 2^{k+1}\right)\right|=0$.

Next notice that the sum

$$
\sum_{K=0}^{\infty} \sum_{n=2^{3^{2 K+j}}}^{2^{3^{2 K+j+1}}} f\left(\frac{\psi(n)}{n}\right) \varphi(n)
$$


must diverge for $j=0$ or 1 . As it will make no difference to the proof, we assume that it diverges for $j=0$ and that

for all $K$.

$$
S \cap\left[2^{3^{2 K+1}}, 2^{3^{2 K+2}}\right)=\emptyset
$$

Now choose integers $0 \leq k \leq \ell \leq 3 k$ for which $S_{k}=S \cap\left[2^{k}, 2^{k+1}\right)$ and $S_{\ell}=S \cap\left[2^{\ell}, 2^{\ell+1}\right.$ ) are both nonempty. For simplicity here and throughout the proof we write $x=2^{k}$ and $y=2^{\ell}$. If $m \in S_{k}$ and $n \in S_{\ell}$ then

$$
\frac{\psi_{k}}{2 x} \leq \Delta(m, n) \leq \frac{2 \psi_{k}}{x},
$$

so in order for equations (6) and (7) to be satisfied we would have to have that

$$
\frac{y \psi_{k}}{2 \sqrt{\log 4 x y}} \leq d \leq 2 y \psi_{k} .
$$

We will establish an upper bound for the number of pairs of integers $m \in S_{k}$ and $n \in S_{\ell}$ which satisfy (13). First of all we note that

$$
\limsup _{n \rightarrow \infty} \frac{\omega(n) \log \log n}{\log n}=1 .
$$

Thus if we suppose that $0<\epsilon_{1}<1 / \log 2-1$ is some fixed constant then we may assume that $x_{0} \in \mathbb{R}$ is chosen large enough so that for all integers $n \geq x_{0}$ we have

$$
\omega(n) \leq \frac{\left(1+\epsilon_{1}\right) \log n}{\log \log n} .
$$

From this it follows that we can find an $\epsilon_{2}>0$ such that

$$
\tau(n) \ll \exp \left(\frac{\left(1-\epsilon_{2}\right) \log n}{\log \log n}\right),
$$

and such that the implied constant is universal. Now suppose that $m \in S_{k}$ and that $d$ is a divisor of $m$ which satisfies (13). Then there at most

$$
4 \psi_{k}^{-1} \sqrt{\log 4 x y}
$$

choices for $n \in S_{\ell}$ which are divisible by $d$. Since $\exp \left\{\left(1-\epsilon_{2}\right) \log x / \log \log x\right\}$ grows faster as $x \rightarrow \infty$ than any power of $\log x$ we have that

$$
\mid\left\{m \in S_{k}, n \in S_{\ell}:(13) \text { holds }\right\}|\ll| S_{k} \mid \psi_{k}^{-1} \exp \left(\frac{\left(1-\epsilon_{2}\right) \log x}{\log \log x}\right) .
$$

Now since $\psi_{\ell} \geq 1 / 2 y$ we use (12) to deduce that

$$
\begin{aligned}
\left|S_{\ell}\right| & \gg\left(\psi_{\ell} \cdot \exp \left(\frac{\left(1 / 2-\epsilon_{2} / 4\right)\left(\log \psi_{\ell}-\log y\right)}{\log \left(\log y-\log \psi_{\ell}\right)}\right)\right)^{-1} \\
& \gg \psi_{k}^{-1} \exp \left(\frac{\left(1-\epsilon_{2} / 2\right) \log y}{\log \log y}\right) .
\end{aligned}
$$


Note that the $k^{2}$ term which appeared in (12) has been absorbed by the extra exponent of $\epsilon_{2} / 4$ here. Inequalities (15) and (16) show that

$$
\mid\left\{m \in S_{k}, n \in S_{\ell}:(\underline{13}) \text { holds }\right\} \mid \ll \frac{\left|S_{k}\right|\left|S_{\ell}\right|}{(\log \log y)^{2}} .
$$

With a view to applying Lemma 2 we have for each nonnegative integer $K$ that

$$
\begin{aligned}
\sum_{2^{3^{2 K}} \leq m, n \leq 2^{3^{2 K+1}}} \lambda\left(\mathcal{E}_{m} \cap \mathcal{E}_{n}\right) & \ll \sum_{3^{2 K} \leq k \leq \ell \leq 3^{2 K}+1} \sum_{\substack{m \in S_{k} \\
n \in S_{\ell}}} \lambda\left(\mathcal{E}_{m}\right) \lambda\left(\mathcal{E}_{n}\right) P(m, n) \\
& \ll \sum_{k, \ell}\left(\sum_{\substack{m \in S_{k} \\
n \in S_{\ell}}}^{\prime} \lambda\left(\mathcal{E}_{m}\right) \lambda\left(\mathcal{E}_{n}\right)+\sum_{\substack{m \in S_{k} \\
n \in S_{\ell}}}^{\prime \prime} \psi(m) \psi(n)\right),
\end{aligned}
$$

where $\sum^{\prime \prime}$ denotes the sum over pairs $m$ and $n$ which satisfy (13) and $\sum^{\prime}$ denotes the sum over pairs which do not. Also in the summand of $\sum^{\prime \prime}$ we have used the fact that

$$
\frac{\varphi(m) \varphi(n) P(m, n)}{n m} \leq 1 .
$$

By using (10) and (17) we find that

$$
\begin{aligned}
\sum_{\substack{m \in S_{k} \\
n \in S_{\ell}}}^{\prime \prime} \psi(m) \psi(n) & \ll \psi_{k} \psi_{\ell} \cdot \mid\left\{m \in S_{k}, n \in S_{\ell}: \text { (13) holds }\right\} \mid \\
& \ll \frac{\psi_{k} \psi_{\ell} \cdot\left|S_{k}\right|\left|S_{\ell}\right|}{(\log \log x)(\log \log y)} \\
& \ll\left(\sum_{m \in S_{k}} \lambda\left(\mathcal{E}_{m}\right)\right)\left(\sum_{n \in S_{\ell}} \lambda\left(\mathcal{E}_{n}\right)\right) .
\end{aligned}
$$

Substituting back into (18) this shows that

$$
\sum_{2^{3^{2 K}} \leq m, n \leq 2^{3^{2 K+1}}} \lambda\left(\mathcal{E}_{m} \cap \mathcal{E}_{n}\right) \ll\left(\sum_{2^{3^{2 K}} \leq n \leq 2^{3^{2 K+1}}} \lambda\left(\mathcal{E}_{n}\right)\right)^{2} .
$$

Finally if $K+1<L$ then

$$
\Delta(m, n) \geq(m n)^{-1 / 2} \text { whenever } m \in\left[2^{3^{2 K}}, 2^{3^{2 K+1}}\right) \text { and } n \in\left[2^{3^{2 L}}, 2^{3^{2 L+1}}\right) .
$$

In this case the Lemma on page 196 of [9] ensures that $\lambda\left(\mathcal{E}_{m} \cap \mathcal{E}_{n}\right) \ll \lambda\left(\mathcal{E}_{m}\right) \lambda\left(\mathcal{E}_{n}\right)$. Putting this together with our results we have for $K \in \mathbb{N}$ that

$$
\sum_{n, m \leq 2^{3^{K}}} \lambda\left(\mathcal{E}_{m} \cap \mathcal{E}_{n}\right) \ll\left(\sum_{n \leq 2^{3^{K}}} \lambda\left(\mathcal{E}_{n}\right)\right)^{2},
$$


and one application of Lemma 2, together with Gallagher's zero-one law, finishes the proof for $\psi$ which satisfy (9).

Step 2. Let us suppose that $\psi$ is any nonnegative arithmetical function for which (2) holds. We will use $\psi$ to define a new function $\psi^{\prime}: \mathbb{N} \rightarrow \mathbb{R}$ as follows. For each $K \in \mathbb{N} \cup\{0\}$, as $n$ runs through the set $S \cap\left[2^{3^{2 K}}, 2^{3^{2 K+1}}\right)$ the range of values taken by $\psi(n)$ falls in the interval $\left[2^{-3^{2 K+1}}, 1 / 2\right)$. Thus we may choose an integer $k=k(K) \in\left\{1, \ldots, 3^{2 K+1}-1\right\}$ so that

$$
\begin{aligned}
\sum_{\substack{2^{2^{2 K}} \\
\psi(n) \in\left[2^{-(k+1)}, 2^{3^{2 K+1}}\right)}} f\left(\frac{\psi(n)}{n}\right) \varphi(n) \geq 3^{-(2 K+1)} \sum_{2^{3^{2 K} \leq n \leq 2^{3^{2 K+1}}}} f\left(\frac{\psi(n)}{n}\right) \varphi(n) \\
\gg \sum_{2^{3^{2 K}} \leq n \leq 2^{3^{2 K+1}}} f\left(\frac{\psi(n)}{n}\right) \frac{\varphi(n)}{\log n} .
\end{aligned}
$$

Then we set

$$
\psi^{\prime}(n)= \begin{cases}\psi(n) & \text { if } n \in\left[2^{3^{2 K}}, 2^{3^{2 K+1}}\right) \text { and } \psi(n) \in\left[2^{-(k(K)+1)}, 2^{-k(K)}\right) \text { for some } K \in \mathbb{N}, \\ 0 & \text { otherwise. }\end{cases}
$$

It is obvious that $W\left(\psi^{\prime}\right) \subseteq W(\psi)$. Furthermore, by the ideas used to prove (16), it follows from (2) and (19) that

$$
\sum_{\substack{n \in \mathbb{N} \\ \psi^{\prime}(n) \neq 0}} \frac{\psi^{\prime}(n) \varphi(n)}{n} \exp \left(\frac{\left(1 / 2-\epsilon_{2} / 8\right) \log \left(\psi^{\prime}(n) / n\right)}{\log \log \left(n / \psi^{\prime}(n)\right)}\right)=\infty,
$$

where $\epsilon_{2}$ is the same as in (14). Since $\psi^{\prime}$ satisfies (9) we can follow almost the exact same argument from the first half of the proof to conclude that $\lambda\left(W\left(\psi^{\prime}\right)\right)=\lambda(W(\psi))=1$. The only difference is that equation (12) will have to be replaced by

$$
\left|\left\{n \in S \cap\left[2^{k}, 2^{k+1}\right)\right\}\right|>\left(k^{2} \psi_{k} \cdot \exp \left(\frac{\left(1 / 2-\epsilon_{2} / 8\right) \log \left(\psi_{k} / x\right)}{\log \log \left(x / \psi_{k}\right)}\right)\right)^{-1} .
$$

However this has no effect on the passage to (16) and thus the rest of the proof remains unchanged.

Remark. In the introduction we mentioned that our Corollary 1 is equivalent to Theorem 3.7(iii) in [8]. In fact, the method used to prove Theorem 3.7(iii) could be used to prove a result which is only slightly weaker than our Theorem 1. Indeed, that this is possible is explicitly mentioned by Harman in [7] in which Theorem 3.7 is first established. However, with our 'direct' approach, not only do we get a slightly stronger result but there is the added advantage that the known limitations in Harman's approach - specifically, see 
Theorem 3.2 in [8] and the comments which succeed it - are no longer necessarily applicable. In other words, our approach can in principle lead to the seriously stronger and more desirable statements outlined in \$5. For example, a sharper upper bound for the quantity

$$
\mid\left\{m \in S_{k}, n \in S_{\ell}:(13) \text { holds }\right\} \mid
$$

would lead to stronger results. The point being highlighted here is that in our estimate (15), we do not even make use of the upper bound imposed on $d$ in (13).

\section{The Mass Transference Principle}

We now turn our attention to establishing Theorem 2. The proof is essentially a nifty application of the Mass Transference Principle introduced in [2]. In a nutshell, the principle allows us to transfer Lebesgue measure statements for lim sup sets to Hausdorff measure statements. The following version of the Mass Transference Principle has been simplified and adapted for the particular application we have in mind. In order to keep the paper self-contained and to introduce useful and necessary notation, it is convenient to start by defining Hausdorff measures $\mathcal{H}^{h}$.

A dimension function $h: \mathbb{R}^{+} \rightarrow \mathbb{R}^{+}$is an increasing, continuous function such that $h(r) \rightarrow 0$ as $r \rightarrow 0$. Suppose $F$ is a non-empty subset of $\mathbb{R}$. For $\rho>0$, a countable collection $\left\{B_{i}\right\}$ of balls in $\mathbb{R}$ with radii $r_{i} \leq \rho$ for each $i$ such that $F \subset \bigcup_{i} B_{i}$ is called a $\rho$-cover for $F$. Define

$$
\mathcal{H}_{\rho}^{h}(F):=\inf \sum_{i} h\left(r_{i}\right)
$$

where the infimum is over all $\rho$-covers of $F$. The Hausdorff $h$-measure of $F$ denoted by $\mathcal{H}^{h}(F)$ is defined as

$$
\mathcal{H}^{h}(F):=\lim _{\rho \rightarrow 0} \mathcal{H}_{\rho}^{h}(F) .
$$

In the case that $h(r)=r^{s}(s \geq 0)$, the measure $\mathcal{H}^{h}$ is the more common $s$-dimensional Hausdorff measure $\mathcal{H}^{s}$. Moreover, the measure $\mathcal{H}^{1}$ is precisely Lebesgue measure $\lambda$. The following easy property

$$
\mathcal{H}^{s}(F)<\infty \quad \Longrightarrow \quad \mathcal{H}^{s^{\prime}}(F)=0 \quad \text { if } s^{\prime}>s
$$

implies that there is a unique real point $s$ at which the Hausdorff $s$-measure drops from infinity to zero (unless the set $F$ is finite so that $\mathcal{H}^{s}(F)$ is never infinite). This point is called the Hausdorff dimension of $F$ and is formally defined as

$$
\operatorname{dim} F:=\inf \left\{s: \mathcal{H}^{s}(F)=0\right\}=\sup \left\{s: \mathcal{H}^{s}(F)=\infty\right\} .
$$

Further details regarding Hausdorff measure and dimension can be found in [6]. We are now in the position to state the Mass Transference Principle.

Given a dimension function $h$, define the following transformation on balls in $\mathbb{R}$ :

$$
B=B(x, r) \mapsto B^{h}:=B(x, h(r)) .
$$


When $h(x)=x^{s}$ for some $s>0$ we also adopt the notation $B^{s}$ for $B^{f}$. Clearly $B^{1}=B$. Recall that $\mathcal{H}^{1}$ is comparable to the one-dimensional Lebesgue measure $\lambda$. Given a sequence of balls $B_{i}, i=1,2,3, \ldots$, as usual its limsup set is

$$
\lim \sup _{i \rightarrow \infty} B_{i}:=\bigcap_{j=1}^{\infty} \bigcup_{i \geq j} B_{i}
$$

For such limsup sets, the following statement is the key to obtaining Hausdorff measure statements from Lebesgue statements.

Theorem 3 (Mass Transference Principle). Let $\left\{B_{i}\right\}_{i \in \mathbb{N}}$ be a sequence of balls in $\mathbb{R}$ with radii $r_{i} \rightarrow 0$ as $i \rightarrow \infty$. Let $h$ be a dimension function such that $x^{-1} h(x)$ is monotonic. For any finite ball $B$ in $\mathbb{R}$, if

$$
\mathcal{H}^{1}\left(B \cap \limsup _{i \rightarrow \infty} B_{i}^{h}\right)=\mathcal{H}^{1}(B)
$$

then

$$
\mathcal{H}^{h}\left(B \cap \limsup _{i \rightarrow \infty} B_{i}^{1}\right)=\mathcal{H}^{h}(B)
$$

For further details and various generalizations of Theorem 3 see [2, 3, 4].

4.1. Proof of Theorem 2 . Given $\epsilon>0$, consider the function $\Psi_{\epsilon}$ defined by

$$
\frac{\Psi_{\epsilon}(n)}{n}:=\left(\frac{\psi(n)}{n}\right)^{s} \quad \text { where } \quad s:=\frac{1-\epsilon}{1+\epsilon} .
$$

Then, in view of the divergent sum condition (41) we have that

$$
\sum_{n \in \mathbb{N}}\left(\frac{\Psi_{\epsilon}(n)}{n}\right)^{1+\epsilon} \varphi(n)=\sum_{n \in \mathbb{N}}\left(\frac{\psi(n)}{n}\right)^{1-\epsilon} \varphi(n)=\infty .
$$

By Corollary 1, it follows that

$$
\mathcal{H}^{1}\left(W\left(\Psi_{\epsilon}\right)\right)=\mathcal{H}^{1}(I) \quad \text { where } I:=[0,1) .
$$

The set $W\left(\Psi_{\epsilon}\right)$ is clearly a limsup set of balls centred at reduced rationals $a / n$ with radii $\Psi_{\epsilon}(n) / n \rightarrow 0$ as $n \rightarrow \infty$. On applying the Mass Transference Principle with $f(r)=r^{s}$, we immediately deduce that

$$
\mathcal{H}^{s}(W(\psi))=\mathcal{H}^{s}(I)=\infty \text {. }
$$

The last equality is valid since $s<1$. It follows from the definition of Hausdorff dimension that

$$
\operatorname{dim}(W(\psi)) \geq s
$$

However, $\epsilon>0$ can be made arbitrarily small and so it follows that $\operatorname{dim}(W(\psi)) \geq 1$. The complementary upper bound is a trivial consequence of the fact that $W(\psi) \subset \mathbb{R}$. 
4.2. Proof of Corollary 2 . We are given that $\sum \psi(n)$ diverges. Without loss of generality, we can assume that

$$
\psi(n)<1 \quad \forall n \in \mathbb{N} .
$$

Otherwise, we define the function $\psi *$ given by $\psi^{*}(n):=\min \{1, \psi(n)\}$. Clearly $\sum \psi^{*}(n)$ diverges and since $W\left(\psi^{*}\right) \subset W(\psi)$ the statement follows on showing that $\operatorname{dim}\left(W\left(\psi^{*}\right)\right)=1$. For any $\epsilon>0$, notice that

$$
\begin{aligned}
\sum_{n \in \mathbb{N}}\left(\frac{\psi(n)}{n}\right)^{1-\epsilon} \varphi(n) \stackrel{\sqrt[21]{>}}{>} \sum_{n \in \mathbb{N}} \psi(n) \frac{\varphi(n)}{n^{1-\epsilon}} \\
\stackrel{81}{\gg} \sum_{n \in \mathbb{N}} \psi(n)=\infty .
\end{aligned}
$$

Thus the divergent sum hypothesis of Theorem 2 is satisfied and the statement of the corollary follows.

\section{Concluding Comments}

A consequence of the Mass Transference Principle is that the Duffin-Schaeffer Conjecture (a Lebesgue measure statement) implies the Generalised Duffin-Schaeffer Conjecture (a Hausdorff measure statement) - see [2] for the details. In particular, the Duffin-Schaeffer Conjecture implies the following weakening of the generalised conjecture.

Conjecture 1. Let $h$ be a dimension function such that $r^{-1} h(r)$ is monotonic and suppose that $r^{-1} h(r) \rightarrow \infty$ as $r \rightarrow 0$. Then

$$
\mathcal{H}^{h}(W(\psi))=\infty \quad \text { if } \quad \sum_{n \in \mathbb{N}} \frac{\psi(n)}{n} \varphi(n)=\infty .
$$

The case when $\mathcal{H}^{h}$ is Lebesgue measure (i.e. when $h(r)=r$ ) is naturally excluded since $\mathcal{H}^{h}(W(\psi)) \leq \mathcal{H}^{h}([0,1))=1$ and the statement is clearly false. Of course, in the Lebesgue case the appropriate statement is the Duffin-Schaeffer Conjecture.

The motivation behind Conjecture 1 is to investigate the Duffin-Schaeffer Conjecture for measures 'arbitrarily' close to Lebesgue measure. Thus, the divergent sum condition remains unchanged but the condition on $h$ means that the measure $\mathcal{H}^{h}$ gives 'extra weight' to sets compared to Lebesgue measure. Heuristically, a ball of radius $r$ is given weight $h(r)$ rather than just $r$. Thus, Conjecture 1 should in principal be easier to establish than the Duffin-Schaeffer Conjecture. Indeed, for any $\epsilon>0$, it is easily seen that Corollary 3 verifies Conjecture 1 for the dimension function $h(r)=r^{1-\epsilon}$. Moreover, let $f$ be as in Theorem 1 and $h$ be any dimension function such that $r^{-1} h(r)$ is monotonic and

$$
\sum_{n \in \mathbb{N}} f\left(h\left(\frac{\psi(n)}{n}\right)\right) \varphi(n)=\infty .
$$


Then Theorem 1 together with the Mass Transference Principle implies that $\mathcal{H}^{h}(W(\psi))=$ $\mathcal{H}^{h}([0,1))$. In particular, this verifies Conjecture 1 for the dimension function

$$
h(r):=r^{1-\frac{1}{1+\log \log 1 / r}} .
$$

Note that this dimension function tends to zero as $r$ tends to zero more slowly than $r$ but more quickly than $r^{1-\epsilon}$ for any $\epsilon>0$. Thus, the next 'significant' and natural step towards the conjecture would be to consider the following problem.

Problem 1. Verify Conjecture 1 for the dimension function $h(r):=r \log \frac{1}{r}$.

Related to Conjecture 1 is the following weakening of the Duffin-Schaeffer Conjecture in which the measure $\lambda$ remains unchanged but 'extra divergence' is assumed.

Conjecture 2. Let $g$ be an increasing function such that $r^{-1} f(r) \rightarrow 0$ as $r \rightarrow 0$. Then

$$
\lambda(W(\psi))=\infty \quad \text { if } \quad \sum_{n \in \mathbb{N}} f\left(\frac{\psi(n)}{n}\right) \varphi(n)=\infty .
$$

In view of the condition imposed on $g$ the above divergence condition implies (11). Thus, Conjecture 2 should in principal be easier to establish than the Duffin-Schaeffer Conjecture. Indeed, Corollary 1 verifies the conjecture for the functions $f(r)=r^{1+\epsilon}$ where $\epsilon>0$ is arbitrary. Moreover, Theorem 11 verifies Conjecture 2 for a function $f$ that tends to zero as $r$ tends to zero faster than $r(-\log r)^{-\epsilon}$ but more slowly than $r^{1+\epsilon}$ for any $\epsilon>0$. For this reason we propose that the following problem represents the next natural step towards Conjecture 2,

Problem 2. Verify Conjecture 2 for the function $f(r):=r\left(\log \frac{1}{r}\right)^{-1}$.

On modifying the argument used to deduce Theorem 2 from Theorem 1 in $\$ 4.1$, it is easily verified that Problem 2 implies Problem 1. Moreover, and just as easily, we see that the Mass Transference Principle enables us to deduce Conjecture 1 from Conjecture 2 whenever $f h(r) \gg r$ for all $r$ sufficiently small.

The overall hope is that investigating the weaker conjectures will yield valuable new insights into the Duffin-Schaeffer Conjecture - a fundamental unsolved problems in metric number theory.

Acknowledgements. We would like to thank Glyn Harman for pointing out the equivalence between our Corollary 1 and his Theorem 3.7(iii) in [8]. SV would like to thank EPSRC for supporting this research - in particular Katharine Bowes who was a great help during the application stage. Also he would like to thank Fernandez, Hobbs and Robinson for inspiring the dynamic duo - Iona and Ayesha - during Year 1. 


\section{REFERENCES}

[1] V. Beresnevich, D. Dickinson and S. L. Velani: Measure Theoretic Laws for limsup Sets, Mem. Amer. Math. Soc. 179 (2006), no. 846, 1-91.

[2] V. Beresnevich and S. L. Velani: A Mass Transference Principle and the Duffin-Schaeffer conjecture for Hausdorff measures, Ann. Math. 164 (2006), 971-992.

[3] _ Schmidt's theorem, Hausdorff Measures and Slicing, IMRN (2006), Article ID 48794, 24 pages.

[4] _ Ubiquity and a general logarithm law for geodesics, Conference proceedings: Dynamical Systems and Diophantine Approximations, Institut Henri Poincar, 7-9 June 2004 (to appear). Pre-print: arXiv:0707.1225.

[5] R. J. Duffin and A. C. Schaeffer: Khintchine's problem in metric Diophantine approximation, Duke Math. J. 8 (1941), 243-255.

[6] K. Falconer: The geometry of fractal sets, Cambridge Tracts in Mathematics, No. 85, Cambridge University Press, 1985.

[7] G. Harman: Some cases of the Duffin and Schaeffer conjecture, Quart. J. Math. Oxford, 241 (1990), 395-404.

[8] G. Harman: Metric number theory, LMS Monographs New Series, vol. 18, Clarendon Press, 1998.

[9] A. D. Pollington and R. C. Vaughan: The k-dimensional Duffin and Schaeffer conjecture, Mathematika 37 (1990), 190-200.

[10] J. D. Vaaler: On the metric theory of Diophantine approximation, Pacific. J. Math., 76, (1978), $527-539$.

Alan K. Haynes: Department of Mathematics, University of York,

Heslington, York, YO10 5DD, England.

e-mail: akh502@york.ac.uk

Andrew D. Pollington: National Science Foundation

Arlington VA 22230 USA

e-mail: adpolling@nsf.ov

Sanju L. Velani: Department of Mathematics, University of York, Heslington, York, YO10 5DD, England.

e-mail: slv3@york.ac.uk 